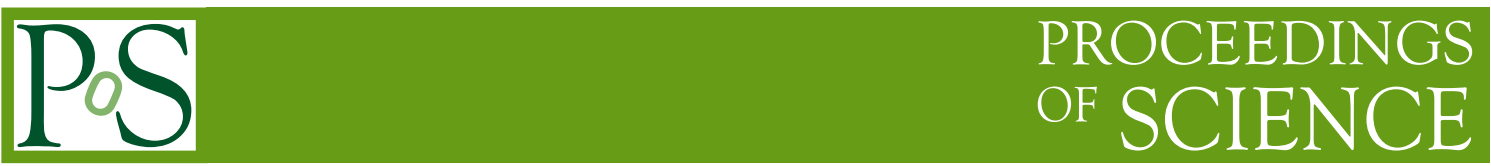

\title{
Muon Collider. A Path to the Future?
}

\section{Daniel Schulte*}

CERN, Geneva, Switzerland

E-mail: daniel.schultedcern.ch

Jean-Pierre Delahaye

CERN, Geneva, Switzerland

\section{Marcella Diemoz}

INFN, Sezione di Roma, Roma, Italy

\section{Ken Long}

Imperial College London

\section{Bruno Mansoulié}

CEA, IRFU, France

\section{Nadia Pastrone}

INFN, Sezione di Torino, Torino, Italy

\section{Lenny Rivkin}

EPFL and PSI, Switzerland

\section{Alexander Skrinsky}

BINP, Russia

\section{Andrea Wulzer}

CERN, Geneva, Switzerland

LPTP, EPFL, Switzerland and University of Padova, Italy

A muon collider would be both a precision and a powerful discovery machine, because it can offer collisions of point-like particles at very high energies, strongly exceeding the energy reach of other lepton colliders. It can even match the discovery of a proton collider with much higher energy, since the muon collision energy is fully available at constituent level unlike for the protons. However, the need for high luminosity faces technical challenges which arise from the short muon lifetime at rest and the difficulty of producing large numbers of muons in bunches with small emittance. Addressing these challenges requires the development of innovative concepts and demanding technologies. In view of the potential importance of muon colliders for the future of high-energy physics it is timely to start an R\&D programme now.

European Physical Society Conference on High Energy Physics - EPS-HEP2019 -

10-17 July, 2019

Ghent, Belgium

\footnotetext{
*Speaker.
} 


\section{Introduction}

Two main muon collider concepts have been developed and proposed: in the first the muons are generated using protons (MAP), in the second using positrons (LEMMA). The proton driven scheme was the object of a well-supported study, mainly in the US, but the coherent effort has now been suspended [1]. The recently proposed positron-driven scheme is being studied with a limited effort mainly at INFN [2]. Since no organised collaboration exists for muon colliders, a small review group has been charged to assess their perspectives and status [3]. This review is based on the material made available by the MAP and LEMMA studies and on some additional calculations.

\section{Physics Goal}

The core goal of a muon collider would be to provide high luminosites at high energies to allow for discoveries and precision physics. Since the cross section for s-channel production scales as $\sigma \propto 1 / s$, the luminosity goal increases with energy. A tentative estimate for the required luminosity is [3]:

$$
\mathscr{L}=\left(\frac{\sqrt{s}}{10 \mathrm{TeV}}\right)^{2} \times 10^{35} \mathrm{~cm}^{-2} \mathrm{~s}^{-1}
$$

This assumes five years of operation. A collision energy of $14 \mathrm{TeV}$ and the corresponding luminosity of $4 \times 10^{35} \mathrm{~cm}^{-2} \mathrm{~s}^{-1}$ would have a discovery potential comparable to FCC-hh.

\section{Proposed Schemes}

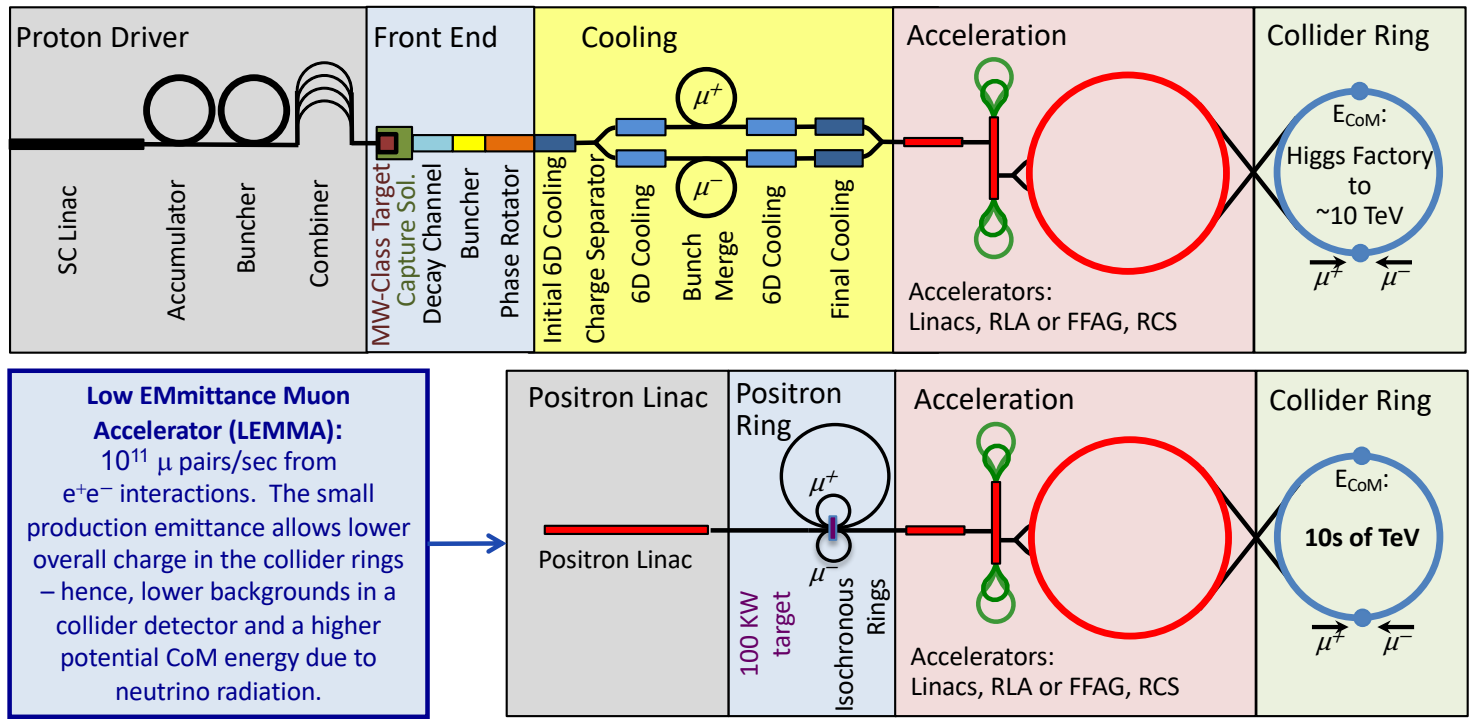

Figure 1: Top: Schematic layout of a potential muon collider with a muon source based on protons. Bottom: Schematic layout of a potential muon collider with a muon source based on positrons.

The proton driven scheme is based on a classical muon production by pion decay. A schematic layout of the MAP scheme is shown in figure 1. An intense proton beam is sent onto a target where 


\begin{tabular}{|lc|c|c|c|}
\hline CoM Energy & $\mathrm{TeV}$ & 1.5 & 3.0 & 6.0 \\
\hline Avg. Luminosity & $10^{34} \mathrm{~cm}^{-2} \mathrm{~s}^{-1}$ & 1.25 & 4.4 & 12 \\
Beam Energy Spread & $\%$ & 0.1 & 0.1 & 0.1 \\
Higgs Production $/ 10^{7} \mathrm{sec}$ & & 37,500 & 200,000 & 820,000 \\
Circumference & $\mathrm{km}$ & 2.5 & 4.5 & 6 \\
Repetition Rate & $\mathrm{Hz}$ & 15 & 12 & 6 \\
$\beta_{x, y}^{\star}$ & $\mathrm{cm}$ & 1 & 0.5 & 0.25 \\
No. muons/bunch & $10^{12}$ & 2 & 2 & 2 \\
Norm. Trans. Emittance, $\varepsilon_{T N}$ & $\mu \mathrm{m}$ & 25 & 25 & 25 \\
Norm. Long. Emittance, $\varepsilon_{L N}$ & $\mathrm{~mm}$ & 70 & 70 & 70 \\
Bunch Length, $\sigma_{s}$ & $\mathrm{~cm}$ & 1 & 0.5 & 0.2 \\
Wall Plug Power & $\mathrm{MW}$ & 216 & 230 & 270 \\
\hline
\end{tabular}

Table 1: Key parameters of a muon collider as taken from the MAP study [1].

it produces a larger number of pions. A fraction of the pions decay into muons that are captured to form muon bunches with positive and negative signs of charge. The phase spaces of these muon beams are very large due to the production process and their lifetimes are limited because of the muon decay. A cooling system reduces the phase spaces rapidly achieving high beam brilliances. In several stages the beams are channeled through matter where the muons loose energy, which reduces the beam phase space, and then re-accelerated. The beams are then accelerated in a combination of linacs and rings to the final energy. In the collider ring they circulate until the muons decay. Examples of key parameters of such a collider are shown in table 1 for different energies.

The MAP study has addressed the global collider parameters and several key technical issues, such as fast-ramping magnets, RF cavities in a high magnetic field, etc. Although it has not reached the level of a Conceptual Design, it is sufficiently comprehensive to give confidence in the collider parameters. Important component and beam tests and an integrated design will be required to fully verify the performance prediction.

In the positron driven scheme, $45 \mathrm{GeV}$ positrons impinging on electrons at rest in a target produce muon pairs close to the reaction threshold, hence with a very low emittance [2]. Therefore no muon beam cooling is required and the beam current can be much smaller than in the MAP scheme mitigating a number of key challenges related to the high muon current in the MAP scheme, as will be discussed below. However, two issues of the original LEMMA design have recently been identified in the review, that potentially reduce the luminosity by orders of magnitude. Currently, the LEMMA team is performing a redesign of the collider concept to address these issues. However, it is too early to assess the results.

Other options of muon production can also be explored. An example is the possible direct production of low-emittance muon beams in a "Gamma Factory", which could also potentially be useful for positron production for the LEMMA scheme. In this scheme, partially stripped heavy ions stored in a ring are collided with a laser pulse to generate intense bursts of X-rays [9]. 

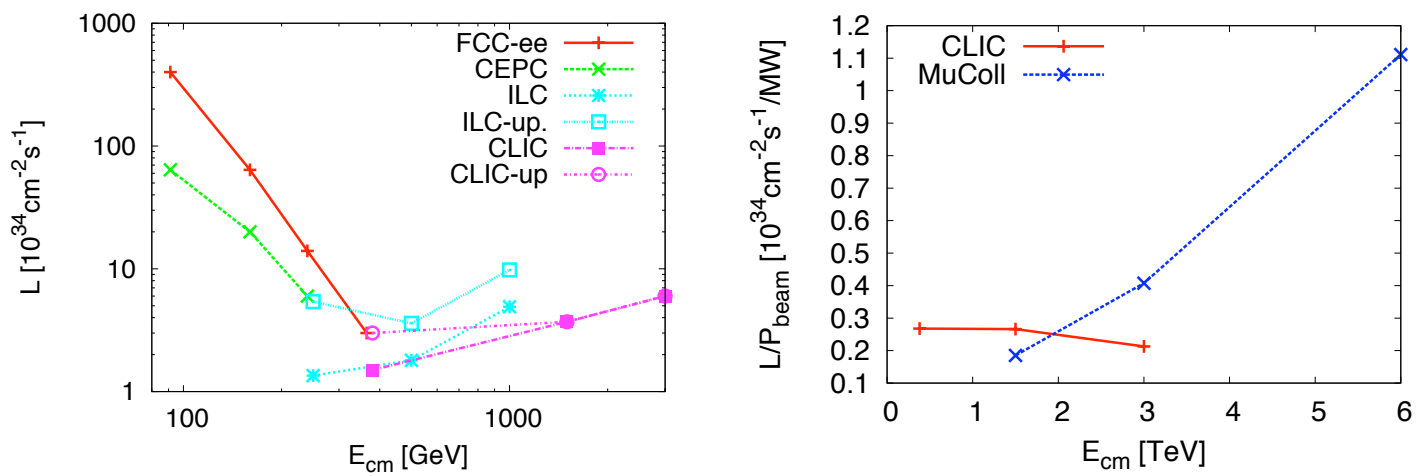

Figure 2: Left-hand side: The luminosities of all experiments combined of FCC-ee, CEPC, ILC and CLIC presented in Granada. Right-hand side: The luminosity per IP per beam power as a function of the centreof-mass energy for CLIC and the proton-based muon collider scheme.

\section{Potential Performance}

Linear electron-positron colliders can also achieve high collision energies and luminosities. The highest centre-of-mass energy proposed in the frame of the Update of the European Strategy for Particle Physics is reached by CLIC [7] with a luminosity of $6 \times 10^{34} \mathrm{~cm}^{-2} \mathrm{~s}^{-1}$. This proposal is based on mature technology. However, the integrated cost of 3 TeV CLIC stage is about 18 GCHF and the power consumption is about $590 \mathrm{MW}$ (generating $28 \mathrm{MW}$ of beam power). The design has not been fully optimised for cost and power but one does not expect important improvements. It therefore seems not obvious that linear colliders can reach higher energies with affordable resources. A muon collider at the $3 \mathrm{TeV}$ energy scale may be competative with CLIC. The tentative power estimate in table 1 hints to a smaller consumption. A cost comparison based on scaling presented in reference [8] indicates that a $6 \mathrm{TeV}$ muon collider could be cheaper than a $3 \mathrm{TeV}$ CLIC. However one has to take this cum grano salis and a conceptual design will be required to confirm or correct the numbers.

A potentially important benefit that muon collider could provide is a linear increase of the luminosity per beam power with increasing collision energy. In contrast, in linear colliders the luminosity per beam power is naturally independent of the collision energy. Figure 2 shows the dependence of the luminosity per beam power for the proton-based muon collider in comparison to CLIC.

In addition, the muon collider can serve more than one collision point. The luminosity spectrum could be substantially better than for a linear electron-positron collider due to the strongly reduced beamstrahlung and initial state radiation.

\section{Challenges and R\&D Status}

The concept is not yet mature enough to be considered for construction today. A strong R\&D programme is needed to develop it to the point where it could be considered as a possible candidate for a high energy physics project. This would be synergetic with other developments, such as high- 
field superconducting magnets, fast-ramping magnets, efficient superconducting RF and normalconducting high-field RF; other topics might also be important such as crystal collimation.

The decay of the accelerated muons drives critical issues:

- At the collision points, the decay electrons induce a large background of electrons and photons. A first simulation study with realistic conditions indicate that this background can be mitigated by suitable shielding, detector design, and analysis, such that it would not damage the physics capability.

- The neutrinos from muon decays along the ring produce showers in the Earth. This leads to some radiation at the location where the plane of the collider ring intercepts the Earth's surface. At very high energies beyond $6 \mathrm{TeV}$, this could ultimately limit the achievable luminosity for the proton-based scheme. The positron-driven scheme would be particularly attractive in this respect since its smaller emittance requires much smaller beam current and thus reduces the neutrino dose, enhancing further the possible energy reach.

The short muon lifetime also defines the challenges for the other systems of the collider as it requires novel beam cooling schemes and rapid acceleration.

A key to the collider luminosity is the generation of brilliant beams and the preservation of their quality. The production of low-emittance muon beams is thus critical, be it as in the LEMMA scheme by generating the beams with a small emittance from the beginning or by cooling beams as in the MAP scheme. A slight cooling of muons in the transverse plane has been demonstrated in MICE $[4,5,6]$. However, a much more substantial cooling in transverse and longitudinal direction remains to be demonstrated.

The alternative LEMMA scheme has to develop a concept that allows to generate a sufficient number of low-emittance muons in single bunches to achieve the luminosity goal.

The complex that accelerates the muons from the source to the collision energy will consist of some combination of linacs and rings. It is an important part of the collider cost and significant study is required to optimise the design and understand the minimum cost.

In the collider ring high field magnets are required to maximise the number of collisions before the muons decay; they also have to sustain the radiation and heat load from the decaying muons.

\section{Recommendations}

The working group concluded the following:

- Muon colliders are not mature enough to be considered for the next project at this moment. But important progress has been made on the proton scheme and new ideas emerged.

- It is important to perform muon collider R\&D. The technology promises the potential to go to very high energies and may be the best option for lepton colliders beyond $3 \mathrm{TeV}$.

- The collider R\&D has strong synergies with other projects. This is true for magnet and RF development and also applies to the physics experiments.

We should not miss this unique opportunity to achieve high-energy lepton collisions. To ensure that the required $R \& D$ is carried out in the most efficient way the group recommends to: 
- Set up an an international collaboration.

- Develop a muon collider concept based on the proton driver scheme and considering existing infrastrucutre.

- Consolidate the positron driver scheme, addressing specifically the target system, bunch combination scheme, beam emittance preservation, acceleration and collider ring issues.

- Carry out an R\&D programme toward the muon collider. Based on the progress of the proton driver and positron-based approaches, develop hardware and research facilities as well as perform beam tests. Preparing and launching an R\&D programme toward a multi$\mathrm{TeV}$ muon collider is mandatory to explore the unique opportunity for high energy physics. A well focused international effort is required in order to exploit existing key competences and to draw the roadmap of this challenging project. The development of new technologies should happen in synergy with other accelerator projects. Moreover, it could also enable novel mid-term experiments.

\section{Potential R\&D Timeline}

The programme could consist of the stages in Fig. 3. In the first stage ( 4 years), the baseline collider concept would be developed. In parallel, a major R\&D project would be defined that addresses the key issues and assesses the solutions. Possibly this project could include some physics goals using high intensity muon or neutrino beams. This phase would require limited resources. The CERN expertise is instrumental and other institutes are willing to join the effort and are already starting. Due to the challenging design issues the project is the perfect environment to exploit new ideas and grow extraordinary skills for the future generation. Depending on the results of the first stage, one can launch the second stage ( 6-years) in which one or more test facilities would be built and operated. The collider design would be further optimised and a conceptual design developed, which includes costing and can be used as the basis for a decision. If the programme is successful, the decision of construction could be taken after a few more years for the preparation of the technical design. If an adequate effort can be put in place, it would be possible to operate a Muon Collider in about 25 years.

The formation of a global collaboration will be essential to carry out the work coherently and efficiently. A strong recommendation from the European Strategy Group would be the decisive starting point for launching this collaboration.

\section{Acknowledgements}

We are pleased to acknowledge the MAP, LEMMA and MICE collaborations for all the excellent developments already achieved on the promising but challenging muon technology, and for all the discussions and useful comments while preparing this document. We particularly thank M. A. Palmer, V. Shiltsev, D. Lucchesi, C. Carli, A. Grudiev, A. Lombardi, G. De Rijk and M. Vretenar. 


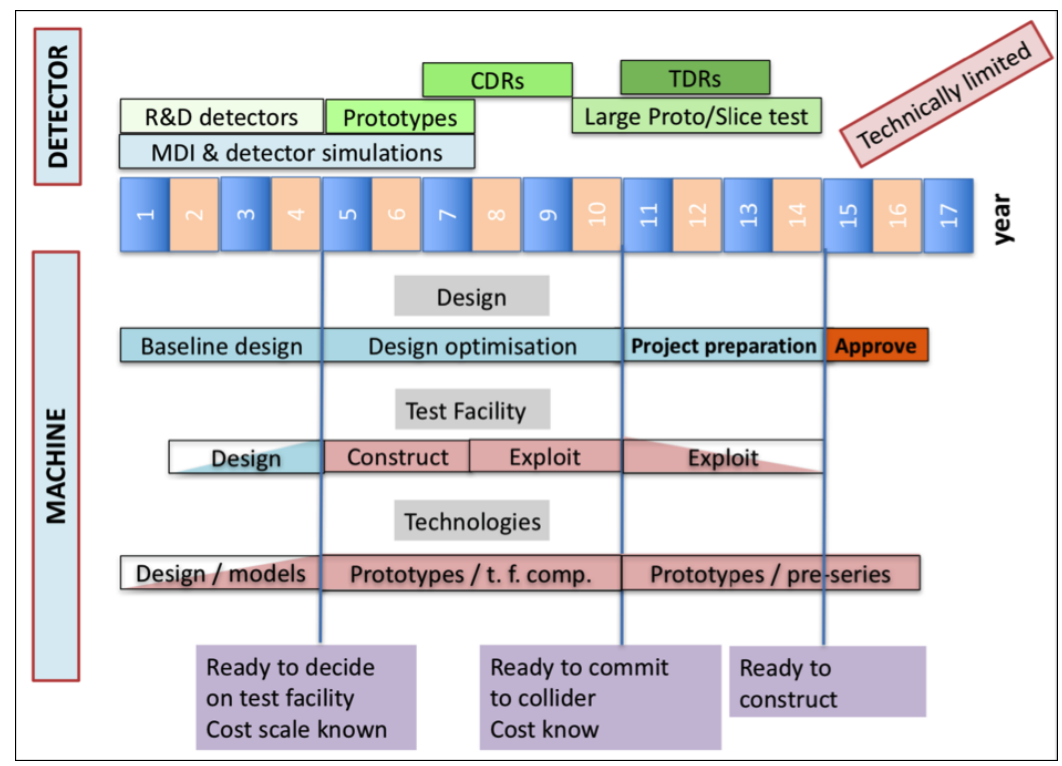

Figure 3: Potential, technically limited, timeline for a muon collider.

\section{References}

[1] M. A. Palmer, "Muon Accelerator Program (MAP)," available online: http://map.fnal.gov.

[2] M. Antonelli, M. Boscolo, R. Di Nardo and P. Raimondi, "Novel proposal for a low emittance muon beam using positron beam on target,” Nucl. Instrum. Meth. A 807 (2016) 101, [arXiv:1509.04454].

[3] Jean-Pierre Delahaye et al., "Muon Colliders", [arXiv:1901.06150]

[4] MICE Collaboration, "Muon Ionization Cooling Experiment (MICE)," available online: http://mice.iit.edu/.

[5] T. A. Mohayai [MICE Collaboration], "First Demonstration of Ionization Cooling in MICE," doi:10.18429/JACoW-IPAC2018-FRXGBE3, [arXiv:1806.01807].

[6] V. Blackmore [MICE Collaboration], "Recent results from the study of emittance evolution in MICE," doi:10.18429/JACoW-IPAC2018-TUPML067, [arXiv:1806.04409].

[7] The CLIC collaboration, CLIC 2018 Summary Report, CERN-2018-005-M, [arXiv:1812.06018]

[8] V. Shiltsev. FERMILAB-CONF-16-536-APC. [arXiv:1705.02011]

[9] See submission ID6 to the European Strategy Update for Particle Physics at https://indico.cern.ch/event/765096/contributions/3295513/contribution.pdf 